\title{
An animal model for checking instrument sterilisation
}

\author{
Evaluation of the effectiveness of decontamination of dental syringes by K. Vickery, A. Pajkos, and Y. Cossart BrDent J \\ 2000; 189: 620-624
}

Aim Steam autoclaving is the gold standard for decontaminating dental instruments, but worldwide disinfection is still widely employed. We have evaluated a range of procedures for their ability to inactivate duck hepatitis B virus contaminating dental syringes.

Methods Residual infectivity of virus suspensions following $2 \%$ glutaraldehyde treatment, ultrasonication or steam sterilisation at $121^{\circ}$ or $134^{\circ}$ was assayed by injecting day-old ducklings and examining their livers for viral DNA 2.5 weeks later. Dental syringes were contaminated with DHBV positive blood, then treated by the same methods. An anaesthetic cartridge containing water was loaded into the syringe and $400 \mu \mathrm{l}$ aliquots used to inject day-old ducklings. Used dental syringes were examined by Scanning Electron Microscopy.

Conclusion Short autoclaving cycles, albeit at raised temperatures, may fail to inactivate the virus because of poor steam penetration, inadequate heat transfer and the accumulation of protective biofilm.

For the full abstract please refer to page 620.

\section{In Brief}

- In vitro assessment of sterilisation and disinfection processes using bacteria as test organisms are the basis of recommendations for clinical practice. It has been difficult to test efficacy against viruses, or to determine the effect of blood and other body fluids.

- We used duck hepatitis $B$ virus which is similar to the human agent to test the efficacy of autoclaving on dental syringes. Low levels of virus (sufficient to infect I/16 ducklings) survived high temperature/short cycle autoclaving of syringes which had been contaminated to mimic 'in use' conditions.

- Electronmicroscopy showed extensive biofilm formation on the luminal surface of used dental syringes, and this protects organisms against destruction by chemical and physical agents. It is also difficult to remove by cleaning.

- We believe that routine cleaning procedures must be rigorous and that clinical guidelines for sterilisation and disinfection should incorporate a significant margin in excess of the conditions based on conventional in vitro tests.

\section{Comment}

Some surgical instruments that are used on dental patients have 'complex narrow channels', 1 into which disinfectants and steam only penetrate with difficulty. Moreover, multiple-use instruments accumulate biofilms of organic residue on their surfaces, and these can protect microorganisms from chemical and physical inactivation. Consequently, sterilisation procedures may fail and infection be spread from patient to patient.

In their article in this issue of the $B D J$, Vickery and colleagues confirm these uncomfortable truths, using an experimental model that is analogous to human hepatitis B virus (HBV) infection. HBV is a virus substantially more resistant to chemical and physical inactivation than the enveloped viruses HIV and hepatitis C virus, though it is not quite as resistant as, for instance, the papilloma (wart) viruses. It is much less resistant than the agents of transmissible spongiform encephalopathy (eg Creutzfeldt-Jakob Disease) for which special sterilisation procedures must be employed. HBV cannot be grown in in vitro cell culture, and the only animal model available for it is the chimpanzee, a threatened higher primate species.

Vickery et al. have made use of duck hepatitis virus (DHV) as a surrogate for $\mathrm{HBV}$ in a series of experiments that investigate the sterilisation of dental instruments. DHV may be present at very high titres in carrier ducks and their blood will infect day old susceptible ducklings when injected intraperitoneally at dilutions of up to $10^{8}$. This infectivity is detected by viral DNA hybridisation tests done on duckling liver 2 weeks later.

With this excellent and very sensitive model, Vickery et al. have looked at several procedures familiar to practising dentists: cleaning in an ultrasonic bath; soaking in $2 \%$ glutaraldehyde; autoclaving at $134^{\circ} \mathrm{C}$ for 3 minutes; and autoclaving at $121^{\circ} \mathrm{C}$ for 15 minutes. Their results do not offer much comfort. They show that for an anaesthetic syringe with a narrow needle lumen neither ultrasonication nor $2 \%$ glutaraldehyde achieve more than very partial inactivation. Pressurised steam sterilisation (ie autoclaving) is far more effective, but it is still not reliable for anaesthetic syringes even though the same autoclaving conditions totally inactivate virus that is more fully accessible to penetration by steam.

While the authors have done enough to justify these conclusions, more experiments will be needed to define precisely what preliminary cleaning, which autoclaves and which autoclaving times and temperatures will inactivate the high titres of HBV still present in the blood of some infected patients after that blood has contaminated dental instruments. For now one can only reiterate that ultrasonication is a cleaning and not a sterilising process, that both pre-cleaning, penetration and sustained contact are essential for glutaraldehyde or steam to act, and that only autoclaving to a correct protocol will reliably sterilise all instruments. It is a crumb of comfort that, for a contaminated instrument, inactivation of at least $10^{6}$ infectious doses of DHV was achieved by Vickery et al using a 15 minute autoclaving cycle. However, this was not always achieved with the shorter cycle at a higher temperature which is likely to be preferred by dental surgeons. In the UK dental autoclaves must meet a British Standard, but dentists would welcome further advice on autoclaves based on appropriate virological tests of their sterilising capacity. This has practical implications, for instance for the number of instrument sets they must equip themselves with.

Vickery and colleagues have devised a way of generating this advice, and it is now up to national regulatory authorities and others with the resources at their disposal to commission evaluations of dental autoclaves based on the model of these authors. As things are, without fully tried and tested sterilising equipment and strict adherence to protocols for its use, dental instruments that have been used on HBV carrier patients could sometimes transmit infection.

1. Vickery K, Pajkos A, and Cossart Y. Evaluation of the effectiveness of decontamination of dental Syringes. Br Dent J 2000; 189: 620-624

\section{Philip P Mortimer}

Director, Sexually Transmitted and Blood Borne Virus Laboratory, Central Public Health

Laboratory, Colindale, London 